\title{
MEASUREMENT OF ERYTHROCYTE MOTIONS IN MICROCHANNELS BY USING A CONFOCAL MICRO-PTV SYSTEM
}

\author{
Rui Lima ${ }^{1,2}$, Takuji Ishikawa', Motohiro Takeda ${ }^{1,3}$, Shuji Tanaka ${ }^{4}$, Yo-suke Imai ${ }^{1}$, Ken-ichi \\ Tsubota', Shigeo Wada ${ }^{5}$, Takami Yamaguchi ${ }^{1}$
}

(1) Dept. Bioeng. \& Robotics, Grad. Sch. Eng., Tohoku Univ., 6-6-01 Aoba, 980-8579 Sendai, Japan.
(2) Dept. Mechanical Tech., ESTiG, Braganca Polyt., C. Sta. Apolonia, 5301-857 Braganca, Portugal.
(3) Div. Surgical Oncology, Grad. Sch. Medicine, Tohoku Univ., 2-1 Seiryo-machi, Aoba-ku, 980-8575

Sendai, Japan.
(4) Dept. Nanomechanics, Grad. Sch. Eng., Tohoku Univ., 6-6-01 Aoba, 980-8579 Sendai, Japan.
(5) Dept. Mechanical Science and Bioeng., Grad. Sch. Eng., Osaka Univ., Toyonaka, 5608531 Osaka, Japan.

\section{INTRODUCTION}

Detailed knowledge on the motion of individual red blood cells (RBCs) flowing in microchannels is essential to provide a better understanding on the blood rheological properties and disorders in microvessels. Several studies on both individual and concentrated RBCs have already been performed in the past $[1,2]$. However, all studies used conventional microscopes and also ghost cells to obtain visible trace RBCs through the microchannel. Recently, considerable progress in the development of confocal microscopy and consequent advantages of this microscope over the conventional microscopes have led to a new technique known as confocal micro-PIV [3, 4]. This technique combines the conventional PIV system with a spinning disk confocal microscope (SDCM). Due to its outstanding spatial filtering technique together with the multiple point light illumination system, this kind of microscope has the ability to obtain in-focus images with optical thickness less than $1 \mu \mathrm{m}$, a task extremely difficult to be achieved by using a conventional microscope.

The main purpose of this paper is to investigate the ability of our confocal micro-PTV system to measure the motion of individual RBCs at different haematocrit (Hct) through microchannels.

\section{MATERIALS AND METHODS}

\subsection{Working fluids and microchannel}

Three working fluids were used in this study: dextran 40 (Dx40) containing about $3 \%$ (3Hct) $14 \%$ (14Hct) and 37\% (37Hct) of human red blood cells (RBCs). The blood was collected from a healthy adult volunteer, where ethylenediaminetetraacetic acid (EDTA) was added to prevent coagulation. The RBCs where separated from the bulk blood by centrifugation (1500 RPM for 10 minutes) and aspiration of the plasma and buffy coat and then washed twice with physiological saline (PS). The washed RBCs were labeled with a fluorescent cell tracker (CM-Dil, C-7000, Molecular Probes) and then diluted with $\mathrm{Dx} 40$ to make up the required $\mathrm{RBCs}$ concentration by volume. All blood samples were stored hermetical at $4^{\circ} \mathrm{C}$ until the experiment was performed at controlled temperature of about $37^{\circ} \mathrm{C}$.

\subsection{Confocal micro-PTV experimental set-up}

The confocal micro-PTV system used in our experiment consists of an inverted microscope (IX71, Olympus) combined with a confocal scanning unit (CSU22, Yokogawa) and a diode-pumped solid state (DPSS) laser (Laser Quantum Ltd) with an excitation wavelength of $532 \mathrm{~nm}$. Moreover, a high-speed camera (Phantom v7.1) was connected into the outlet port of the CSU22. The microchannel was placed on the stage of the inverted microscope where the flow rate of the working fluids was kept constant $(\mathrm{Re}=0.004)$ by means of a syringe pump (KD Scientific Inc.). A thermo plate controller (Tokai Hit) was set to $37^{\circ} \mathrm{C}$

All the confocal images were captured with a resolution of $640 \times 480$ pixels, at a rate of 100 frames/s with an exposure time of 9.4 $\mathrm{ms}$. The recorded images were transfered to the computer and then evaluated in the Image $\mathrm{J}(\mathrm{NIH})$ [8] by using the manual tracking MTrackJ [9] plugin. As a result it was possible to track single RBCs through the middle plane of the microchannel $(75 \mu \mathrm{m}$ in diameter). Deatailed information about the experimental set-up, used in the present study, has already been described previously [6]. 


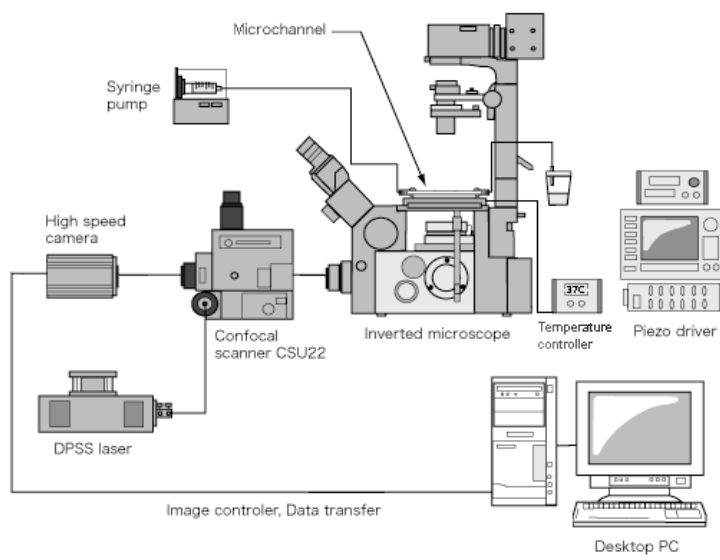

Figure 1. Confocal micro-PTV experimental set-up.

\section{RESULTS AND DISCUSSION}

\subsection{Tracking displacement of RBCs at different Hcts}

By using the optical sectioning ability of the confocal system it was possible to obtain series of optical sectioned images at the middle of microchannel. Figure 2 shows images with both RBCs (halogen illumination) and labeled RBCs (laser-emitted light) at different Hcts together with the correspondent time position tracking of individual RBCs.
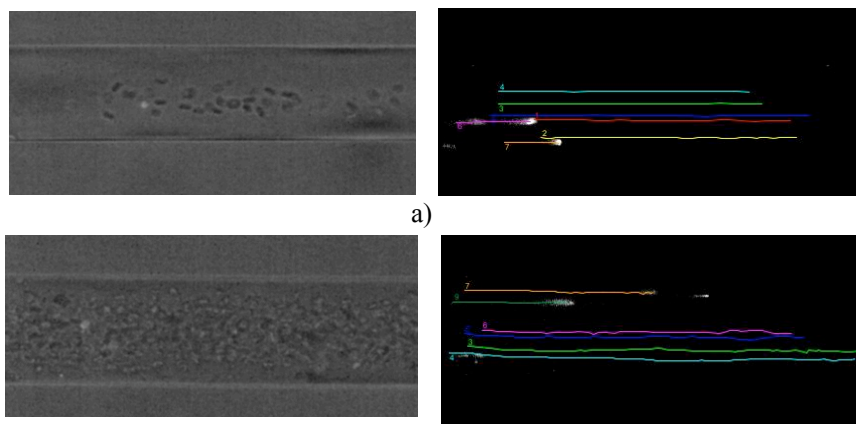

b)
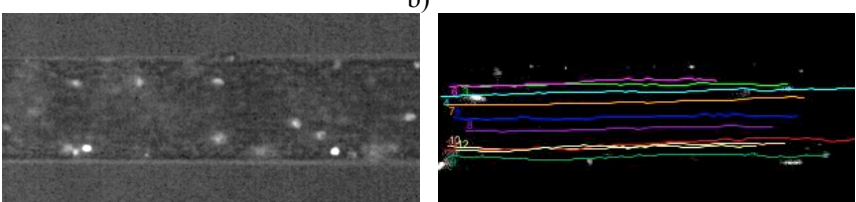

c)

Figure 2. Both normal and labeled RBCs (bright spots) with a) $3 \%$ Hct, b) $14 \%$ Hct, c) $37 \%$ Hct (20x, 1.6 zoom objective lens) and correspondent RBC paths displacement.

Figure 2 show the ability of the confocal system to track single RBCs at the middle plane with Hct up to $37 \%$. Furthermore it also is possible to observe that at $3 \% \mathrm{Hct}$ the $\mathrm{RBC}$ paths are almost parallel to the flow direction without any appreciable fluctuations on the radial direction. By contrast, at $14 \%$ and $37 \%$ Hct the RBC paths exhibit erratic radial displacements due the high-concentration of RBCs on the adjacent streamlines. These results show clearly that this technique can provide detailed information about micro-scale disturbance effects caused by $\mathrm{RBCs}$ to the blood flow.

\subsection{Diffusion coefficient at different Hcts}

A diffusion coefficient $\left(\mathrm{D}_{\mathrm{rr}}\right)$ to quantify the radial displacement of the tracer RBCs has been defined as $[2,5]$ :

$$
D_{r r}=\lim _{t \rightarrow \infty} \frac{\left\langle\left[r_{r}(t)-r_{r}(0)\right]\right\rangle^{2}}{2 t}
$$

where $\mathrm{r}$ and $\mathrm{t}$ are radial displacement and time interval respectively.

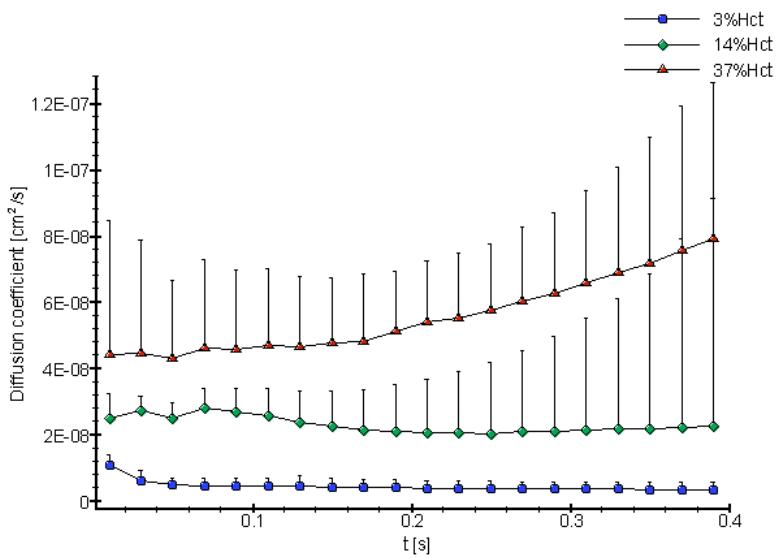

Figure 3. Diffusion coefficient in a circular PDMS microchannel ( $75 \mu \mathrm{m}$ in diameter).

The preliminary results shown in Figure 3, suggests that the RBC paths are strongly dependent on the Hct and as a result the radial RBC diffusivity increases with the heamatocrict. An ongoing detailed study to calculate the radial $\mathrm{RBC}$ diffusivity at 100 and $50 \mu \mathrm{m}$ microchannels is currently under way.

\section{REFERENCES}

1. Goldsmith, H., 1971, "Red cell motions and wall interactions in tube flow", Federation Proceedings, Vol.30, 5, pp. 1578-1588.

2. Goldsmith, H. and Marlow J., 1979, "Flow behavior of erythrocytes. II. Particles motions in concentrated suspensions of ghost cells", Journal of Colloid and Interface Science, Vol.71, 2, 383-407.

3. Lima, R., Wada, S., Tsubota, K. and Yamaguchi, T., 2006 "Confocal micro-PIV measurements of three dimensional profiles of cell suspension flow in a square microchannel", Meas. Sci. Tech., Vol.17, pp. 797- 808.

4. Lima, R., Wada, S., Takeda, M., Tsubota, K., Yamaguchi, T., 2007, "In vitro confocal micro-PIV measurements of blood flow in a square microchannel: the effect of the haematocrit on instantaneous velocity profiles", J. Biomech., (accepted).

5. Haile, J., 1992, Molecular Dynamics Simulation: elementary methods, John Wiley and Sons, USA.

6. Abramoff, M., Magelhaes, P., and Ram, S., 2004, "Image Processing with ImageJ, Biophotonics International”, Vol.11, 7, pp. 36-42.

7. Meijering, E., Smal, I., and Danuser, G., 2006, "Tracking in Molecular Bioimaging,", IEEE Signal Processing Magazine, Vol.23, 3, pp. 46-53. 